\title{
Qianggan extract improved nonalcoholic steatohepatitis by modulating InCRNA/ circRNA immune ceRNA networks
}

\author{
Mingzhe Zhu ${ }^{1,2+}$, Meng $\mathrm{Li}^{1+}$, Wenjun Zhou', Yang Yang ${ }^{3}$, Fenghua $\mathrm{Li}^{3}$, Li Zhang ${ }^{1 *}$ and Guang $\mathrm{Ji}^{1 *}$ (D)
}

\begin{abstract}
Background: The traditional Chinese medicine prescription, Qianggan formula have been confirmed to be effective on non-alcoholic steatohepatitis (NASH), however, the underlying molecular mechanisms remain obscure.

Methods: Thirty-six male C57BL/6 mice were randomly divided into three groups: normal chow diet group; methionine-and-choline-deficient diet (MCD) group, and Qianggan extract (QG) intervention group $(0.4 \mathrm{~g} / \mathrm{kg}$ daily) that fed with MCD. The efficacy of QG was biochemically and histologically evaluated. The expression profiles of messenger ribonucleic acids (mRNAs), long non-coding RNAs (IncRNAs) and circular RNAs (circRNAs) were examined using microarray and verified by RT-qPCR.

Results: QG significantly improved the phenotypic characteristics of NASH, as serum alanine aminotransferase (ALT), aspartate aminotransferase (AST), alkaline phosphatase (ALP), and lactate dehydrogenase (LDH) levels and liver inflammatory cytokines were significantly decreased. By the cutoff of a 1.5-fold change and $P<0.05$, 6193 mRNAs, 5692 IncRNAs and 4843 circRNAs were identified as differentially expressed between the MCD and normal groups, and 514 mRNAs, 1182 IncRNAs and 443 circRNAs were identified as differentially expressed between the QG and MCD groups. The intersections (244 mRNAs, 259 IncRNAs and 98 circRNAs) among the three groups were chosen for analysis. Gene Ontology (GO) terms and Kyoto Encyclopedia of Genes and Genomes (KEGG) pathway enrichment revealed that most overlapping mRNAs were related to immune functions such as natural-killercell-mediated cytotoxicity, intestinal immune network for IgA production, and T cell receptor signaling pathway. Pathway interactions, protein-protein interactions and molecular complex detection (MCODE) analysis identified numerous immune-related hub genes e.g. natural cytotoxicity triggering receptor 1(Ncr1), C-X-C motif chemokine ligand 9 (Cxc19), Klra1, and Cd28. Finally, two IncRNAs (Sngh1 and Slc36a3os) and four circRNAs (circ_0009029, circ_ 0004572, circ_0009212 and circ_0009453) in competing endogenous RNA (ceRNA) networks were constructed by Cytoscape, and immune-related mRNAs (e.g., Cd28, Cd8a, II15, and Klrk1) were involved in the ceRNA networks.
\end{abstract}

Conclusions: LncRNA and circRNA-associated immune ceRNA networks might be the targets of QG in alleviating $\mathrm{NASH}$, and our work may provide valuable clues for exploring the mechanisms underlying the effect of QG.

Keywords: Non-alcoholicsteatohepatitis, IncRNA, circRNA, ceRNA, Microarray, Bioinformatics

\footnotetext{
*Correspondence: zhangli.hl@163.com; jiliver@vip.sina.com

${ }^{\dagger}$ Mingzhe Zhu and Meng Li contributed equally to this work.

${ }^{1}$ Institute of Digestive Diseases, Longhua Hospital, Shanghai University of

Traditional Chinese Medicine, No.725 South Wanping Road, Shanghai

200032, China

Full list of author information is available at the end of the article
}

(c) The Author(s). 2019 Open Access This article is distributed under the terms of the Creative Commons Attribution 4.0 International License (http://creativecommons.org/licenses/by/4.0/), which permits unrestricted use, distribution, and reproduction in any medium, provided you give appropriate credit to the original author(s) and the source, provide a link to the Creative Commons license, and indicate if changes were made. The Creative Commons Public Domain Dedication waiver (http://creativecommons.org/publicdomain/zero/1.0/) applies to the data made available in this article, unless otherwise stated. 


\section{Background}

Nonalcoholic fatty liver disease (NAFLD) refers to a broad spectrum of diseases characterized by fat infiltration in the liver [1]. Non-alcoholic steatohepatitis (NASH) is a progressive form of NAFLD characterized by lobular inflammation, hepatocellular ballooning, and fibrosis with an inherent risk of progression to end-stage hepatocellular carcinoma [2, 3]. Twenty percent of NASH patients are reported to develop cirrhosis, and $30-40 \%$ of patients with NASH cirrhosis die from liver-related diseases, NASH has become the third most common indication for liver transplantation in the United States [4].

The pathogenesis of NASH has not been fully elucidated, and the mechanisms appear to be multifactorial. "Two hits" and "multiple hits" hypotheses have been proposed to describe the pathogenesis of NASH $[5,6]$. Insulin resistance, inflammatory cytokines, and oxidative stress are thought to be important in the progression of NASH. Pharmacological agents such as insulin sensitizers, antioxidants, and lipid-lowering drugs have been implicated to improve NASH characteristics and progression [7]. However, there is no consensus regarding the effective and appropriate drug therapy for NASH.

Accumulating evidence suggests that traditional Chinese medicine formulae are promising agents for treating NASH [8]. The Qianggan formula was designed according to the theory of traditional Chinese medicine, contains 16 ingredients: Artemisia scoparia Waldst. \& Kitam., Isatis tinctoria L., Angelica sinensis(Oliv.)Diels., Paeonia lactiflora Pall., Salvia miltiorrhiza Bunge., Curcuma wenyujin Y.H.Chen et C.Ling, Astragalus membranaceus(Fisch.) Bunge., Codonopsis pilosula(Franch.)Nannf., Alisma orientale(Sam.)Juz., Polygonatum kingianum Collett \& Hemsl., Rehmannia glutinosa (Gaertn.) DC., Dioscorea oppositifolia L., Crataegus pinnatifida Bunge., Medicated Leaven Massa Medicata Fermentata, Gentiana macrophylla Pall., and Glycyrrhiza uralensis Fisch., and has been reported as an effective and safe remedy for the treatment of NAFLD/ NASH [9]. Protecting hepatocytes is proposed; however, the molecular mechanisms underlying the efficacy are still unclear.

Coding ribonucleic acids (RNAs) coupled with noncoding (nc) RNAs are involved in NASH pathogenesis [10]. MicroRNAs (miRNAs), long non-coding RNAs (lncRNAs) and circular RNAs (circRNAs) are different species of ncRNAs [11]. MiRNAs are 18-25 nucleotides long, evolutionarily conserved, single-stranded RNA, that negatively modulate the expression of their target mRNAs [12]. Numerous miRNAs have been reported to be correlated with NASH $[13,14]$. LncRNAs are RNA transcripts $>200$ nucleotides, that can regulate gene expression through many patterns, including chromosome remodeling, and transcriptional and post-transcriptional processing. LncRNAs are involved in the pathogenesis of liver diseases and have potential diagnostic, prognostic, and therapeutic importance [15], and contribute to the development of NASH and fibrosis [10, 16]. LncRNAs can act as competing endogenous RNAs (ceRNAs) or miRNA sponges, interacting with miRNAs in a manner that sequesters these molecules and reduce their regulatory effect on target mRNAs [17]. Similar to IncRNAs, circRNAs (a class of RNA molecules that lack $5^{\prime}-3^{\prime}$ ' ends and poly A tails and covalently form closed loops) can also function as ceRNAs or miRNA sponges [18]. The importance of ceRNAs has been addressed in various diseases. A deep RNA sequencing study constructed comprehensive circRNA-associated ceRNA networks, and inferred the diagnosis and therapy of Alzheimer's disease [19]. Another study provided a lncRNA-related ceRNA network and suggested two-lncRNA signatures as independent biomarkers for the prognosis of lung squamous cell carcinoma [20].

To systemically understand the efficacy of Qianggan extract (QG) on NASH, a comprehensive microarray analysis of mRNAs, lncRNAs and circRNAs was conducted to obtain expression profiles. By identifying differentially expressed mRNAs, lncRNAs and circRNAs and constructing ceRNA networks, our data shed new light on potential novel biomarkers and drug targets of NASH.

\section{Methods}

\section{Preparation of Qianggan extract}

The Qianggan formula was composed of 16 ingredients (Table 1), and the extract was prepared using the following procedures: all of the ingredients were soaked in 10 times the volume of water and boiled for $2 \mathrm{~h}$, the supernatant were collected, 8 times volume of water was added and the mixture was boiled another $1.5 \mathrm{~h}$. Then, the supernatant was collected, 6 times the water volume was added, and the mixture was and boiled $1.5 \mathrm{~h}$ for the third time. All the supernatants were filtered, the $\mathrm{PH}$ was adjusted to 8 . The extract powder of was then obtained by concentrating the solution to a density ratio of 1.35 .

\section{Animal treatment and sample collection}

Six-week-old male C57BL/6 mice were obtained from Shanghai Laboratory Animal Co. Ltd., and maintained in a specific pathogen-free environment with temperatureand humidity-controlled conditions. Thirty-six mice were allocated to six per cage, and randomly divided into three groups ( $n=12$ per group) based on bodyweight: the normal group, fed a chow diet; the methionine-andcholine-deficient diet (MCD) group, fed a MCD diet; and the QG group, fed a MCD diet and supplemented with the extract (Orient Pharmaceutical Co., Ltd., 0.4 g $/ \mathrm{kg}$ daily). The extract was dissolved in distilled water 
Table 1 The composition of Qianggan formula

\begin{tabular}{llll}
\hline Scientific name & Chinese Name & Pharmaceutical portion & Percent \\
\hline Artemisia scoparia Waldst. \& Kitam. & Yin-Chen & Cauline leaf & $11 \%$ \\
Isatis tinctoria L. & Ban-Lan-Gen & Root & $5.5 \%$ \\
Angelica sinensis(Oliv.)Diels & Dang-Gui & Root & Root \\
Paeonia lactiflora Pall. & Bai-Shao & Rhizome & $5.5 \%$ \\
Salvia miltiorrhiza Bunge. & Dan-Shen & Rhizome & $11 \%$ \\
Curcuma wenyujin Y.H.Chen et C.Ling & Yu-Jin & Root & $5.5 \%$ \\
Astragalus membranaceus(Fisch.)Bunge & Huang-Qi & Root & $11 \%$ \\
Codonopsis pilosula(Franch.)Nannf. & Dang-Shen & Tuber & $5.5 \%$ \\
Alisma orientale(Sam.)Juz. & Ze-Xie & Rhizome \\
Polygonatum kingianum Collett \& Hemsl & Huang-Jing & Root \\
Rehmannia glutinosa (Gaertn.) DC. & Shen-Di & Rhizome \\
Dioscorea oppositifolia L. & Shan-Yao & Fructus \\
Crataegus pinnatifida Bunge. & Shan-Zha & Zymotic product \\
Medicated Leaven Massa Medicata Fermentata & Liu-Shen-Qu & Root & $5.5 \%$ \\
Gentiana macrophylla Pall & Qin-Jiao & Rhizome & $4.5 \%$ \\
Glycyrrhiza uralensis Fisch. & Gan-Cao & & $4.4 \%$ \\
\hline
\end{tabular}

and intragastrically administered ( $1 \mathrm{ml} / 100 \mathrm{~g}$ body weight) for 4 consecutive weeks, and the other mice were given an equal volume of distilled water. Body weight was recorded once per week. At the end of the experiment, mice were weighed at 8 am the next morning and anesthetized by intraperitoneal injection with $2 \%$ pentobarbital sodium (1.5 $\mathrm{ml} / \mathrm{kg}$ bodyweight). Then, blood was collected by cardiac puncture, centrifuged at $3000 \mathrm{rpm}$ for $10 \mathrm{~min}$ at $4{ }^{\circ} \mathrm{C}$, and serum was separated. Liver tissue was quickly removed, rinsed with $0.9 \%$ sodium chloride solution and weighed. Liver tissues from the identical lobe and position were cut and then fixed in $10 \%$ neutral-buffered formalin. The remainder was stored at $-80^{\circ} \mathrm{C}$ after snap freezing in liquid nitrogen. After sample collection, the animals were physically euthanized by quickly breaking the spines to avoid insufficient anesthetization, and bodies were disposed of by the central department of animal care.

All animal procedures were approved by the Animal Experiment Ethics Committee of Shanghai University of Traditional Chinese Medicine (approval number: 201703014).

\section{Determination of the serum enzymes levels}

Serum alanine aminotransferase (ALT), aspartate aminotransferase (AST), alkaline phosphatase (ALP), and lactate dehydrogenase (LDH) were analyzed using the Hitachi full-automatic system with the corresponding kits (Wako, Richmond, VA, USA).

\section{Histology staining}

Liver samples were fixed in 10\% formalin for $48 \mathrm{~h}$, embedded in paraffin, sectioned to $4 \mu \mathrm{m}$ thickness, stained with hematoxylin and eosin (H\&E), and examined under a light microscope at $200 \times$ magnification.

\section{Microarray detection and analysis RNA preparation and microarray detection}

Liver total RNA was extracted using TaKaRa RNAiso Plus regent and RNA integrity was assessed using an Agilent Bioanalyzer 2100 (Agilent Technologies, Santa Clara, CA, US). RNA was further purified by an RNeasy mini kit (QIAGEN, Hilden, Germany) and RNase-Free DNase Set (QIAGEN), amplified and labeled by Low Input Quick Amp WT Labeling Kit (Agilent Technologies), purified and hybridized with a SBC-Mouse ceRNA microarray (SBC, Shanghai, China). Nine slides were scanned by an Agilent Microarray Scanner with default settings: dye channel, green; scan resolution, $3 \mu \mathrm{m}$; PMT 100\%; 20 bit. Data were extracted with Feature Extraction version 10.7 (Agilent Technologies). Raw data were normalized by the Quantile algorithm, limma packages in $\mathrm{R}$.

\section{Microarray data processing}

The statistical significance of differentially expressed mRNAs, lncRNAs and circRNAs among groups (MCD vs normal and QG vs MCD) was identified by the cutoff of 1.5-fold change and $P<0.05$. The intersections of differentially expressed mRNAs, lncRNAs and circRNAs between MCD vs normal and QG vs MCD were determined by Venn diagrams. The overlapped mRNAs, lncRNAs and circRNAs were subjected to hierarchical clustering by Cluster version 3.0 to generate an overview of the characteristics of expression profiles. 


\section{Gene ontology (GO) and pathway analysis}

To explore the main biological function of differentially expressed genes, functional enrichment was performed based on GO and Kyoto Encyclopedia of Genes and Genomes (KEGG) database [21, 22]. GO classifies gene functions according to molecular function, biological process and cellular component. Pathway enrichment analysis revealed the main metabolic and signaling pathways. The enriched pathways interaction networks were constructed using the Cytoscape plug-in ClueGO and CluePedia.

\section{Protein-protein interaction (PPI) network analysis}

PPI network analysis was performed based on the STRING database (https://string-db.org/). The network was visualized by Cytoscape, and hub genes were obtained by screening the degree of connectivity of each node in the network. In the network, nodes represent genes and lines represent the interactions. To identify the significant modules in the network, Cytoscape plugin MCODE (Molecular Complex Detection) was conducted with a score $>4$.

\section{LnCRNA and circRNA associated ceRNA network construction}

According to the ceRNA hypothesis, ceRNA members can compete for the same miRNA response elements (MREs) to regulate each other. Various types of RNA transcripts compete with one another for binding to a shared miRNA, thereby negatively regulating miRNAmediated gene silencing [23]. Hence, the expression pattern of mRNAs should be in the same direction as lncRNAs or circRNAs. The potential miRNA-binding sites were searched by the sequences of IncRNAs, circRNAs and mRNAs based on the TargetScan database (http:// www.targetscan.org/). Then, lncRNA-miRNA-mRNA and circRNA-miRNA-mRNA networks were constructed based on lncRNA/circRNA-miRNA and miRNA-mRNA regulation pairs. In addition to overlapping miRNA binding, mRNAs with an expression pattern in the same direction as lncRNAs or circRNAs were filtered out to construct lncRNA/circRNA -miRNA-mRNA ceRNA networks by Cytoscape.

\section{Quantitative real-time PCR assay}

To validate our microarray data, quantitative Real-time experiments were performed as previously described [24]. We selected 13 representative RNAs to verify the expression, and primers sequences were listed in Table 2.

\section{Statistical analysis}

Data were expressed as mean \pm SD and were analyzed by one-way analysis of variance by SPSS version 18.0. $P<$ 0.05 was considered significant different.
Table 2 Sequences of the primers used for PCR

\begin{tabular}{|c|c|c|}
\hline Genes & Forward primer & Reverse primer \\
\hline Cdc14a & TGCACTACACCTCTITCGACC & $\begin{array}{l}\text { AGGAGGGTTTGAGCC } \\
\text { AGACA }\end{array}$ \\
\hline Cnr2 & $\begin{array}{l}\text { ATGGCCGTGCTCTATATTATC } \\
\text { CT }\end{array}$ & $\begin{array}{l}\text { ATGGTCACACTGCCG } \\
\text { ATCTTC }\end{array}$ \\
\hline $\mathrm{Cd} 28$ & CTGTTCTTGGCTCTCAACTTCTT & $\begin{array}{l}\text { GCTGACCTCGTTGCTA } \\
\text { TCTACC }\end{array}$ \\
\hline$\| 18 \mathrm{r} 1$ & ACTACTCCTGCGTGTITTCTGTC & $\begin{array}{l}\text { CATCCTITCCTAGTTCT } \\
\text { ACACCAAC }\end{array}$ \\
\hline Klrc1 & CTCGCAGCTCCATTTCAGTC & $\begin{array}{l}\text { CAATTAAGACAAAACA } \\
\text { GATGAGGC }\end{array}$ \\
\hline Ner1 & ATGCTGCCAACACTCACTG & $\begin{array}{l}\text { ATGATGGGTTTCGGGA } \\
\text { GAGTC }\end{array}$ \\
\hline $\mathrm{Ccl} 8$ & TCTACGCAGTGCTTCTTTGCC & $\begin{array}{l}\text { AAGGGGGATCTTCAG } \\
\text { CTITAGTA }\end{array}$ \\
\hline Rian & GGTGCTGCCTCAGTCTIT & TCCAGGATTGATTGTGCT \\
\hline Foxd2os & $\begin{array}{l}\text { ATCGGTAGTGGAAATCTG } \\
\text { TAAG }\end{array}$ & $\begin{array}{l}\text { TCAAAGCGACTGTATT } \\
\text { AGGC }\end{array}$ \\
\hline Snhg1 & TCATGTTGTCACAGCACC & $\begin{array}{l}\text { GCCCTTTACACTTG } \\
\text { GAG }\end{array}$ \\
\hline Circ_0007379 & AGACTGCCAGCCCCTCATC & $\begin{array}{l}\text { GGGAAGCACTCTGGAT } \\
\text { GTTAGG }\end{array}$ \\
\hline Circ_0004572 & $\begin{array}{l}\text { CATCAATGAAGTCAAGCCT } \\
\text { ACAGAG }\end{array}$ & $\begin{array}{l}\text { CATGGACCTCGTACCCTT } \\
\text { TCTC }\end{array}$ \\
\hline GAPDH & GTGCCGCCTGGAGAAACC & $\begin{array}{l}\text { GGTGGAAGAGTGGGAG } \\
\text { TTGC }\end{array}$ \\
\hline
\end{tabular}

\section{Results}

Phenotypic characteristics

Four weeks of MCD feeding induced typical NASH features, and H\&E staining showed significant hepatic steatosis and inflammation in MCD mice compared to normal mice (Fig. 1h). Serum AST (Fig. 1d), ALT (Fig. 1e), ALP (Fig. 1f) and LDH (Fig. 1g) levels were all markedly increased in MCD mice. QG extract supplementation improved hepatic steatosis and inflammation, and restored increased serum parameters (Fig. 1d-g). The body weight and liver weight were decreased in the MCD mice in comparison to normal mice, while QG had no effect on these parameters (Fig. 1a\&b). The ratio of liver weight and body weight among the groups revealed no significant difference (Fig. 1c).

Differentially expressed mRNAs, IncRNAs and circRNAs

By the cutoff of a 1.5-fold change and $P<0.05,6193$ differentially expressed mRNAs (differentially expressed genes, DEGs), 5692 differentially expressed lncRNAs (DElncRNAs) and 4843 differentially expressed circRNAs (DEcircRNAs) were identified in the MCD vs normal group. There were 514 DEGs, 1182 DElncRNAs and 443DEcicRNAs in QG vs MCD group. By Venn diagram, overlapping 244 DEGs (Fig. 2a), 259 DElncRNAs (Fig. 2b) and 98DEcircRNAs (Fig. 2c) were filtered out 


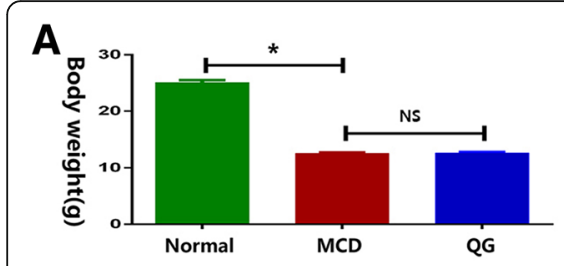

D

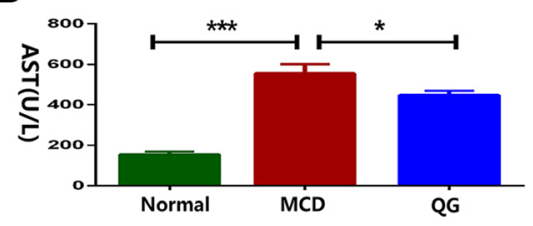

G

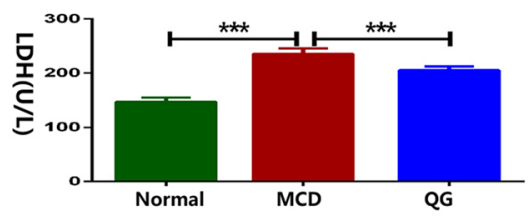

B

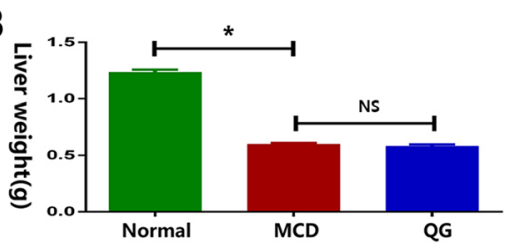

E

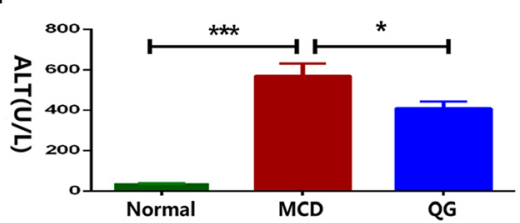

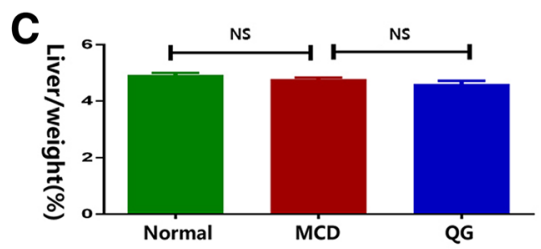

$F$

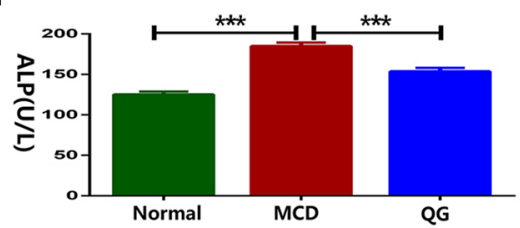

H

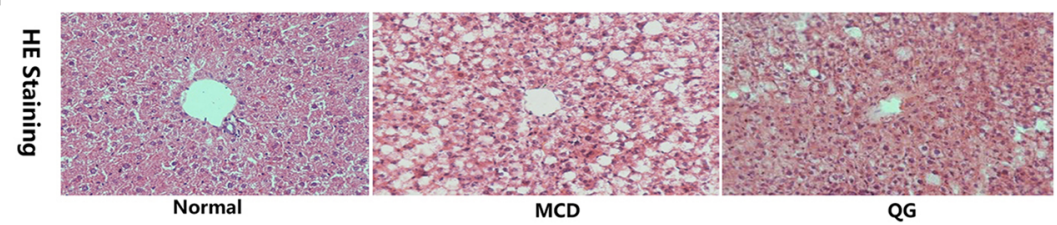

Fig. 1 Phenotypic characteristics of the mice. a Body weight. b Liver weight. c Liver/body weight ratio. d Serum AST. e Serum ALT. f Serum ALP. g Serum LDH. h Liver sections stained with H\&E. Data were represented as mean $\pm \mathrm{SD}, n=12$ per group, $^{\mathrm{a}} P<0.05,{ }^{\mathrm{b}} P<0.01$

and chosen for further analysis. Representative overlapping DEGs, DElncRNAs and DEcircRNAs are listed in Tables 3, 4 and 5, respectively. Detailed information of differentially expressed RNAs was listed in Additional file 1: Table S1, Additional file 2: Table S2 and Additional file 3: Table S3.

To obtain an overview of the expression pattern of DEGs, DElncRNAs and DEcircRNAs among groups, hierarchical clustering was performed. Most of the DEGs, DElncRNAs and DEcircRNAs exhibited opposite expression patterns between MCD vs normal and QG vs MCD (Fig. 2d-f). For instance, the DEGs Cxcl9, Cd28, Cd8a, Btla, Klra1, Klrk1 and Ncr1were up-regulated in the MCD vs normal group, but down-regulated in the QG vs MCD group. The DElncRNAs Snhg1 and Slc36a3os were increased in the MCD vs normal group, but decreased in the QG vs MCD group. DEcircRNAscirc_0009029 and circ_0004572 were induced in the MCD vs normal group, but reduced in the QG vs MCD group. These results indicated that Qianggan extract could affect the gene expression of NASH mice.

\section{GO and pathway analysis}

To further explore the main biological functions of DEGs, GO and pathway enrichments were performed (Additional file 4: Table S4). The 244 overlapping DEGs were enriched in immune-related GO terms and KEGG pathways such as natural killer cell-mediated cytotoxicity, intestinal immune network for IgA production, and T-cell receptor signaling pathways. Figure $3 \mathrm{a}$ and $\mathrm{b}$ lists the top 30 enriched GO terms and KEGG pathways. To identify the key genes in the pathways, enriched KEGG pathway interactions were constructed, and hub genes such as Cd28, Klra1, and Klra7 were indicated (Fig. 3c).

\section{PPI network analysis}

PPI network analysis and MCODE were conducted to depict interactions and significant modules among the overlapped 244 DEGs. A PPI network with 118 nodes (average of 2.66 neighbors) was obtained (Fig. 4a). The important hub genes with no less than five neighbors were identified. By MCODE, two significant modules were filtered out with a score $>4$. Most hub genes, such as Cd28, Cd8a, Cxcl9, Klka1, Klrk1and Ncrlc, and the modules were immune related.

LncRNA and circRNA associated immune ceRNA networks LncRNAs and circRNAs interacting as ceRNAs play important roles in regulating gene expression. However, the biological functions of most lncRNAs and circRNAs are unknown. To unravel the functions of identified DElncRNAs and DEcircRNAs, we constructed ceRNA networks. Interactions of miRNAs with mRNAs, lncRNAs and circRNA were predicted based on the Targetscan database (Additional file 5: Table S5). By combining of literature retrieval and the ceRNA hypothesis, we constructed two lncRNA- and four circRNA-associated immune ceRNA networks that were implied in NASH.

LncRNA Snhg1 was a ceRNA of eight miRNAs (e.g., miR-199a-3p, miR-490-3p, and miR-412-3p) that targeted 74 mRNAs (e.g., $C d 28, C d 8 a$, and Cxcl9) (Fig. 5a). LncRNA Slc36a3os was a ceRNA of four miRNAs (miR324-3p, miR-680, miR-3473b and miR-3102-5p.2-5p) that targeted 66 mRNAs (e.g., Cd28, Il18rap, and Il15) 


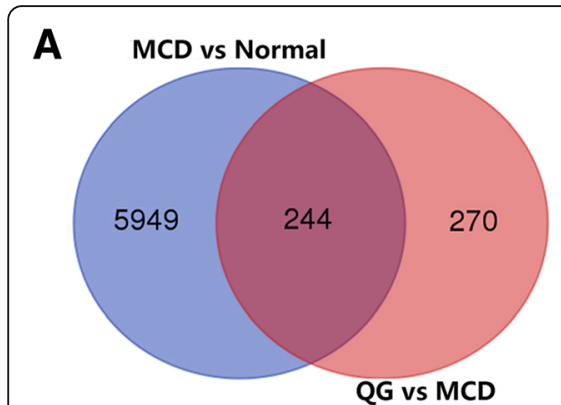

D
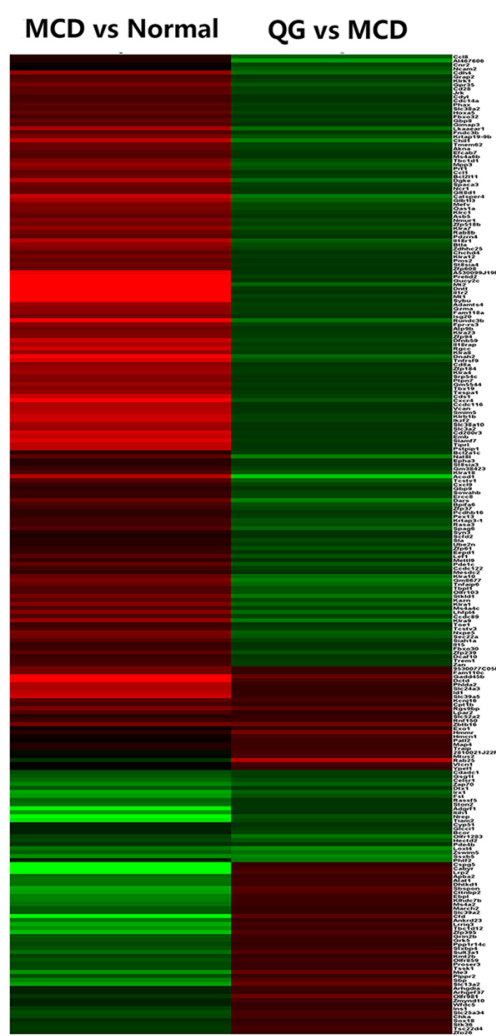

mRNAs
B

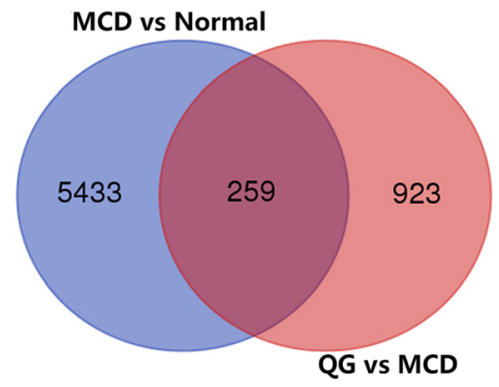

E

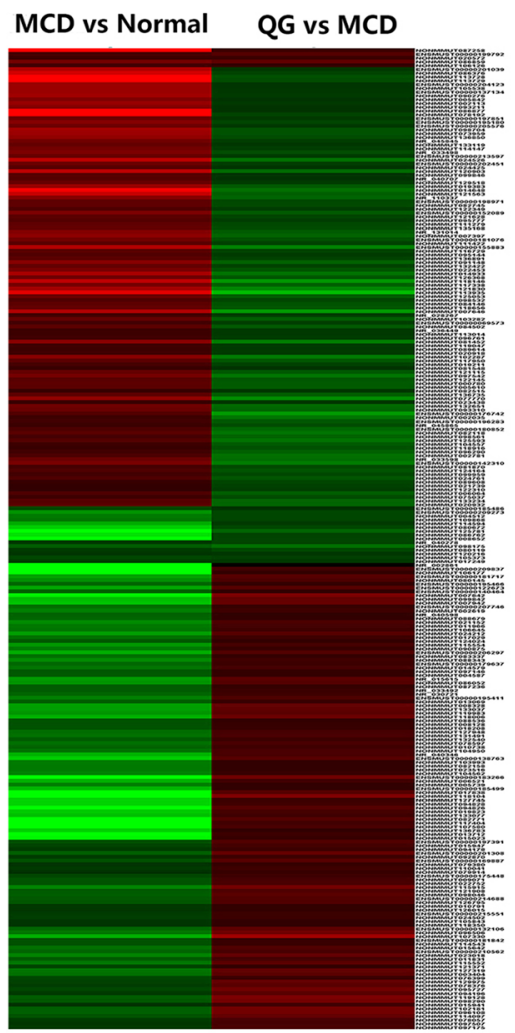

IncRNAs

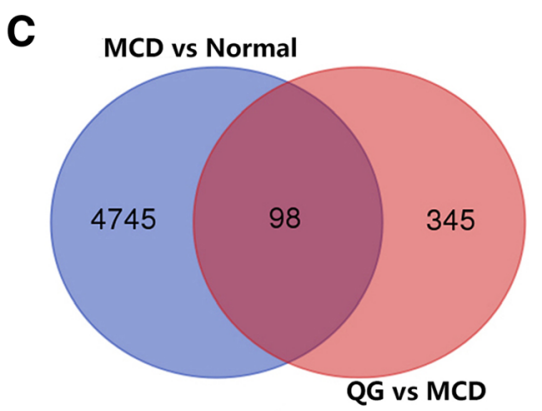

F

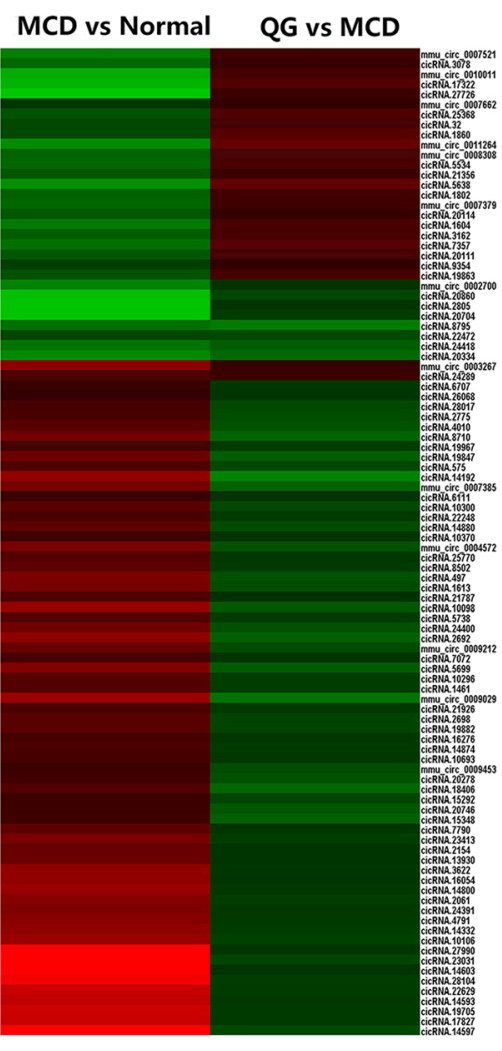

CircRNAs

Fig. 2 Hierarchical cluster of overlapped DEGs, DEIncRNAs and DEcircRNA. a Venn diagram of overlapping DEGs. b Venn diagram of overlapping DElncRNAs. c Venn diagram of overlapping DEcircRNAs. $\mathbf{d}$ Heatmap of overlapping DEGs. e Heatmap of overlapping DElncRNAs. $\mathbf{f}$ Heatmap of overlapping DEcircRNAs. Gene expression is represented by colors, with brighter red for higher values and brighter green for lower values

(Fig. 5b). LncRNA Snhg1 and Slc36a3os shared 53 mRNAs such as $C d 28, C d 8 a$, and Klrk1 (Fig. 5c \&f). Moreover, the GO terms revealed that mRNAs involved in lncRNASngh1 or Slc36a3os ceRNA networks were enriched in immune processes (Fig. 5d, e\&g).

In addition, circ_0004572 was a ceRNA of 2 miRNAs that targeted 32 mRNAs (Fig. 6a). Circ_0009453 was a ceRNA of four miRNAs that targeted 54 mRNAs (Fig. 6c). Circ_0009212 was a ceRNA of 4 miRNAs that targeted 68 mRNAs (Fig. 6e). Circ_0009029 was a ceRNA of miR-362$5 p$ that targeted 24 mRNAs (Fig. 6g). GO analysis demonstrated that mRNAs involved in the networks were enriched in immune-related terms (Fig. 6b, d, f and h). We obtained circRNA-associated immune ceRNA networks that comprised 4 circRNAs, 11 miRNAs and $100 \mathrm{~m}$ RNAs, and we also noticed that the four circRNAs shared four mRNAs (Fig. 6i and j).

\section{Validation of the RNA expression}

To validate our findings based on microarray data, we selected 13 RNAs for verification by RT-qPCR. Fold changes of RNAs (MCD vs normal and QG vs MCD) were calculated based on the average expression values. As shown in Fig. 7, most of the results from RT-qPCR 
Table 3 Representative overlapped differentially expressed genes

\begin{tabular}{lllll}
\hline GeneSymbol & $\begin{array}{l}\text { MCD vs Con } \\
\text { Fold change }\end{array}$ & $\begin{array}{l}\text { MCD vs Con } \\
P \text {-value }\end{array}$ & $\begin{array}{l}\text { QG vs MCD } \\
\text { Fold change }\end{array}$ & $\begin{array}{l}\text { QG vs MCD } \\
\text { P-value }\end{array}$ \\
\hline Ccl8 & 2.44 & 0.03 & 0.25 & 0.03 \\
Ssxb5 & 0.31 & 0.00 & 0.33 & 0.01 \\
Cd28 & 2.10 & 0.00 & 0.59 & 0.02 \\
Gm8677 & 2.76 & 0.01 & 0.36 & 0.00 \\
Chil1 & 4.73 & 0.03 & 0.36 & 0.02 \\
Catsper4 & 5.35 & 0.04 & 0.37 & 0.02 \\
Stkld1 & 2.61 & 0.01 & 0.37 & 0.02 \\
Klrk1 & 2.15 & 0.04 & 0.59 & 0.02 \\
Olfr1283 & 0.60 & 0.03 & 0.38 & 0.01 \\
Cdh4 & 3.42 & 0.04 & 0.41 & 0.00 \\
Left1 & 2.04 & 0.03 & 0.50 & 0.01 \\
Cd8a & 3.15 & 0.01 & 0.65 & 0.04 \\
Cabyr & 0.09 & 0.00 & 1.73 & 0.01 \\
Cxcl9 & 2.09 & 0.00 & 0.64 & 0.15 \\
II15 & 1.62 & 0.02 & 0.65 & 0.02 \\
II18r1 & 4.44 & 0.05 & 0.46 & 0.05 \\
Sbp & 0.34 & 0.04 & 2.02 & 0.05 \\
Rgs9bp & 2.78 & 0.04 & 2.12 & 0.02 \\
Gadd45b & 9.64 & 0.04 & 2.22 & 0.03 \\
Zbtb16 & 2.69 & 0.01 & 2.58 & 0.02 \\
\hline & & & &
\end{tabular}

were in consistence with microarray data, which might suggest the reliability of our microarray analysis and findings.

\section{Discussion}

Here we confirmed the effect of QG on NASH mice, and comprehensive microarray analysis revealed a batch of differentially expressed mRNAs, IncRNAs and circRNAs. Through bioinformatics analysis, we constructed lncRNA- circRNA-associated immune ceRNA networks, and obtained certain immune-related biological processes, pathways and hub genes that responded QG intervention.

Qianggan formula was initially designed for treat hepatitis B virus in China, and it showed obvious effect in reducing plasma ALT, AST and inflammatory parameters [25]. In clinical trials of NAFLD, Qianggan formula treatment significantly reduced hepatic lipid content in patients, as evidenced by ultrasound test. In addition, plasma TG and ALT levels were decreased upon treatment [26]. In high-fat diet-induced NAFLD rats, 4 weeks of QG treatment prevented leptin resistance, and alleviated lipid accumulation and inflammation in the liver [27]. Because the formula is a mixture of 16 ingredients and undoubtedly has multiple targets, integrative highthroughput analysis could facilitate the exploration of
Table 4 Representative differentially expressed IncRNAs

\begin{tabular}{lllll}
\hline GeneSymbol & $\begin{array}{l}\text { MCD vs Con } \\
\text { fold change }\end{array}$ & $\begin{array}{l}\text { MCD vs } \\
P \text {-value }\end{array}$ & $\begin{array}{l}\text { QG vs MCD } \\
\text { fold change }\end{array}$ & $\begin{array}{l}\text { QG vs MCD } \\
\text { P-value }\end{array}$ \\
\hline Snhg1 & 2.47 & 0.02 & 0.63 & 0.00 \\
Slc36a30s & 4.24 & 0.04 & 0.35 & 0.03 \\
RP24-175C20.19 & 0.61 & 0.05 & 1.62 & 0.01 \\
RP23-62D21.4 & 0.49 & 0.00 & 1.98 & 0.00 \\
RP23-368 K22.5 & 2.31 & 0.04 & 0.61 & 0.05 \\
Rian & 0.09 & 0.02 & 2.47 & 0.00 \\
Gm45609 & 0.03 & 0.00 & 1.74 & 0.00 \\
Gm45414 & 0.43 & 0.00 & 1.84 & 0.01 \\
Gm45204 & 0.24 & 0.01 & 1.97 & 0.05 \\
Gm44427 & 3.51 & 0.02 & 0.65 & 0.00 \\
Gm43823 & 0.51 & 0.03 & 1.97 & 0.04 \\
Gm43814 & 2.39 & 0.01 & 0.63 & 0.05 \\
Gm43168 & 4.24 & 0.00 & 0.65 & 0.04 \\
Gm43065 & 2.19 & 0.02 & 0.55 & 0.01 \\
Gm42640 & 1.81 & 0.02 & 1.99 & 0.02 \\
Gm42599 & 0.46 & 0.01 & 2.26 & 0.05 \\
Gm38351 & 0.43 & 0.02 & 1.55 & 0.02 \\
Gm37570 & 0.35 & 0.01 & 1.91 & 0.04 \\
Gm3716 & 1.73 & 0.04 & 0.48 & 0.01 \\
Gm37143 & 2.92 & 0.01 & 0.57 & 0.01 \\
Gm28875 & 0.55 & 0.01 & 0.66 & 0.02 \\
Gm26645 & 2.53 & 0.05 & 0.52 & 0.03 \\
Gm25694 & 0.56 & 0.02 & 1.66 & 0.04 \\
Gm25545 & 0.26 & 0.01 & 2.01 & 0.02 \\
Gm20633 & 1.98 & 0.04 & 0.28 & 0.05 \\
Gm20052 & 2.39 & 0.01 & 0.60 & 0.02 \\
\hline & & & &
\end{tabular}

Table 5 Representative overlapped differentially expressed circRNAs

\begin{tabular}{lllll}
\hline circBase_ID & $\begin{array}{l}\text { MCD vs Con } \\
\text { fold change }\end{array}$ & $\begin{array}{l}\text { MCD vs Con } \\
P \text {-value }\end{array}$ & $\begin{array}{l}\text { QG vs MCD } \\
\text { fold change }\end{array}$ & $\begin{array}{l}\text { QG vs MCD } \\
P \text {-value }\end{array}$ \\
\hline circ_0011264 & 0.32 & 0.01 & 2.22 & 0.02 \\
circ_0010011 & 0.24 & 0.00 & 1.80 & 0.03 \\
circ_0009453 & 1.64 & 0.01 & 0.53 & 0.01 \\
circ_0009212 & 2.35 & 0.04 & 0.54 & 0.02 \\
circ_0009029 & 3.56 & 0.02 & 0.38 & 0.02 \\
circ_0008308 & 0.44 & 0.03 & 1.76 & 0.02 \\
circ_0007662 & 0.64 & 0.01 & 1.54 & 0.00 \\
circ_0007521 & 0.35 & 0.01 & 1.68 & 0.03 \\
circ_0007385 & 2.59 & 0.01 & 0.45 & 0.01 \\
circ_0007379 & 0.43 & 0.03 & 1.68 & 0.03 \\
circ_0004572 & 2.69 & 0.03 & 0.52 & 0.05 \\
circ_0003267 & 3.11 & 0.01 & 1.56 & 0.04 \\
circ_0002700 & 0.34 & 0.00 & 0.66 & 0.03 \\
\hline
\end{tabular}




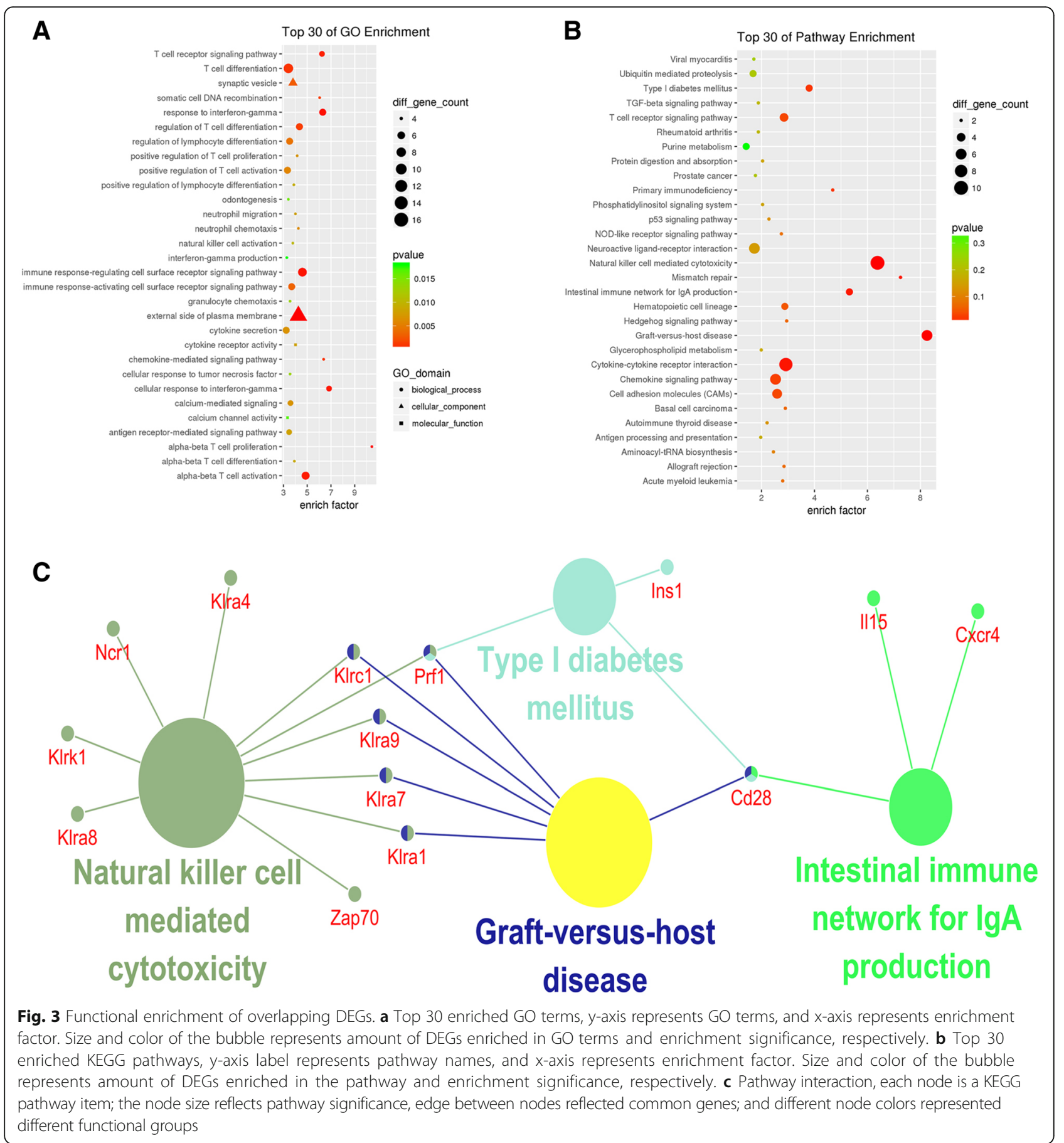

the underlying mechanisms, and the ceRNA networks we have reported may provide valuable information.

Immune responses are involved in the development of NASH [28]. Many types of innate (NK cells, NKT cells, and kupffer cells/macrophages) and adaptive ( $\mathrm{T}$ cells and $B$ cells) immune cells are enriched in the liver and play pivotal roles in NASH progression [29]. NK- cellmediated cytotoxicity and the T-cell receptor signaling pathway are important immune processes, and studies have shown that the dysfunction of these pathways is involved in liver inflammation. Accordingly, we identified changes in several immune hub genes, such as $C d 28$, Cd8a, Cxcl9, Klra1, Klrk1, Klra9, Il15, and Ncr1that related to the effect of Qianggan extract.

LncRNAs might have important roles in NAFLD/ NASH [30]. LncRNA SNHG1 is up-regulated by $M Y C N$ amplification and could be a potential prognostic biomarker for neuroblastoma intervention in patients [31]. 


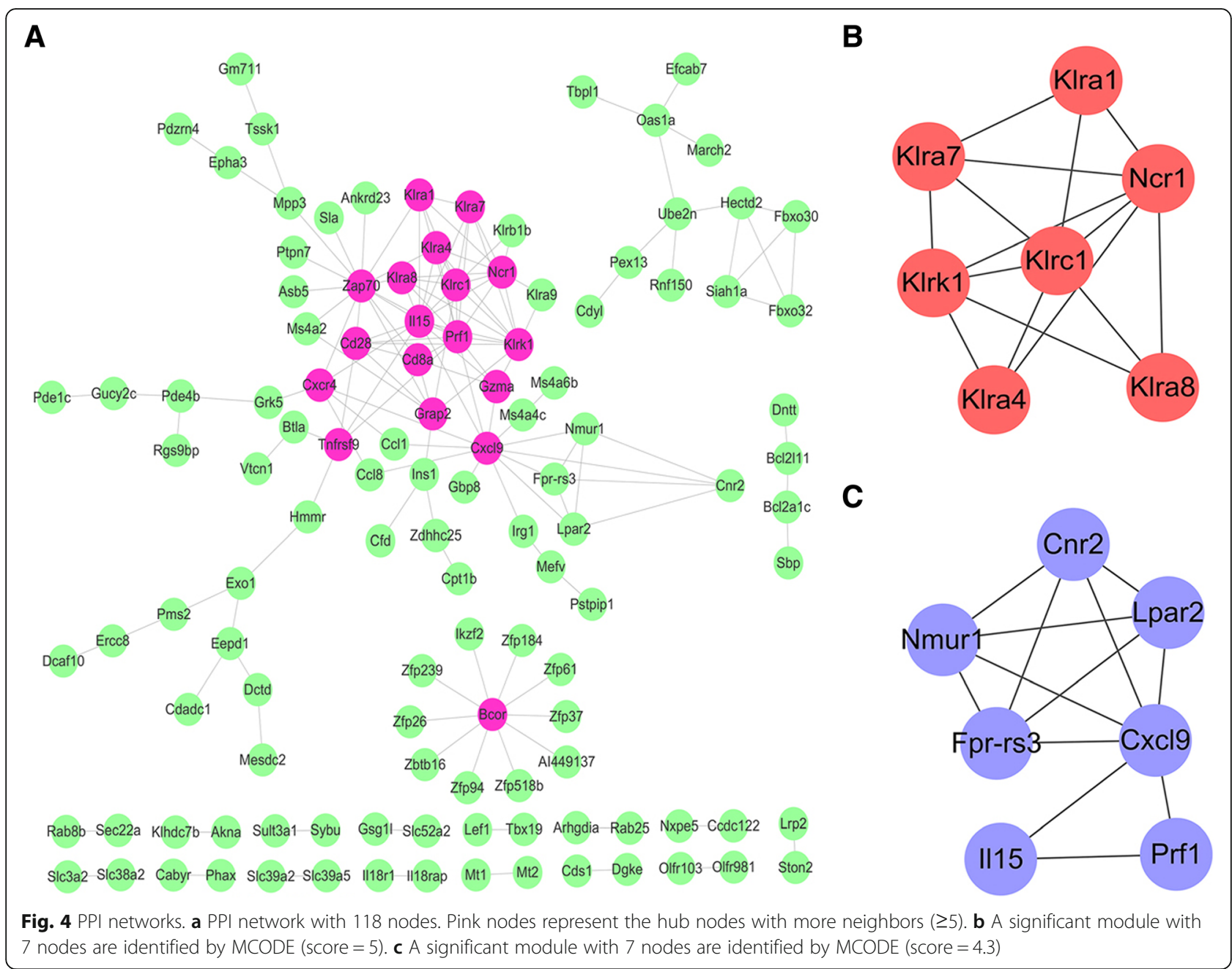

Another study reported that decreased IncRNASNHG1 inhibits colorectal carcinoma tumorigenesis and might act as an important potential therapeutic target [32]. Of note, lncRNA Snhg1 is reported to be increased in hepatocellular carcinoma [33]. In the present study, we observed that the level of lncRNASnhg1was increased in NASH, whereas it was decreased by Qianggan extract. Considering the association between $\mathrm{NASH}$ and hepatocellular carcinoma, lncRNASnhg1 might be a therapeutic target of QGE and a potential biomarker of NASH.

According to the ceRNA hypothesis, IncRNAs act as miRNA sponges to negatively regulate miRNAs and positively regulate target mRNAs. A recent study revealed that lncRNA SNHG1 acted as a ceRNA of miR326 targeted NOB1 [34]. A previous report showed that lncRNA SNHG1 serves as a ceRNA to negatively regulate miR-199a-3p and enhance $C D K 7$ expression, which might be a potential therapeutic target for prostate cancer [35]. We noticed that lncRNA Snhg1 serves as a
ceRNA of miR-199a-3p, and 7 other miRNAs that targeted a series of immune-related mRNAs. CD28 is a membrane protein belonging to the family of costimulatory molecules that synergistically acts as a second signal with the T-cell receptor complex [36]. Researchers have revealed that deletion of CD28 establishes a new pro/anti-inflammatory balance, and protects the liver against steatosis [37]. In accordance with previous studies, we observed that $C d 28$ mRNA expression was elevated in NASH and reduced after QGE treatment in mice. CD8A is a constituent of the CD8 antigen found on most cytotoxic $\mathrm{T}$ cells and acts as a coreceptor with the T-cell receptor [38]. Here, we noticed that $C d 8 a$ was up-regulated in NASH mice but downregulated after Qianggan treatment, suggesting $C d 8 a$ as a potential therapeutic target for NASH. CXCL9 is secreted by monocyte-derived dendritic cells within the liver and is a pro-inflammatory cytokine that attracts Th1 and Th17 cells to the liver [39]. CXCL9 was elevated in patients with autoimmune hepatitis and mice 


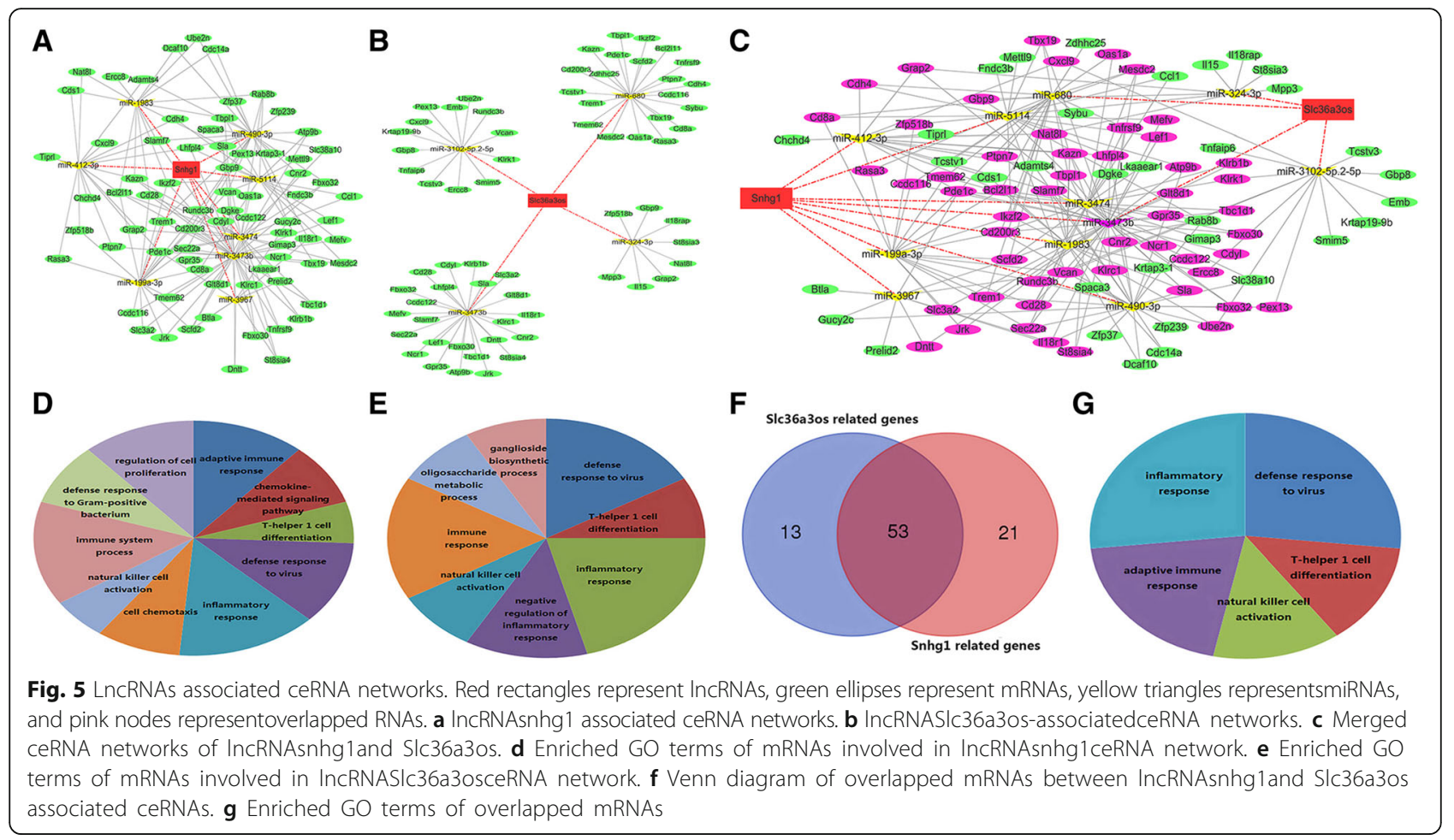

with fatty liver [40, 41], indicating that CXCL9 inhibition is a potential strategy in NAFLD treatment.

MiR-324-3p is associated with malignant clinicopathologic features and poor prognosis of hepatocellular carcinoma [42]. In the present study, we noticed that miR-324-3p-targeted mRNAs were differentially expressed among the groups. IL-15 has been implicated in high-fat-diet-induced hepatic lipid accumulation and inflammation through immune regulation [43]. Researchers have analyzed the level of IL-15 in patients with acute hepatic failure and have suggested that overexpression of IL-15 might cause liver injury in humans [44]. Similar to lncRNAs, circRNAs can also act as ceRNAs to inhibit miRNAs and positively regulate associated mRNAs. We identified several circRNAs acting as ceRNAs in NASH. MiR-760-3p has been implicated in immunological processes and miR5099 in liver injury $[45,46]$. Intriguingly, we observed that 32 targeted mRNAs were enriched in immune related functions and several mRNAs (e.g., Ccll and Il18r1) were related to liver inflammation and fibrosis. Inhibition of the CC chemokine ligand CCL1 has been suggested as a successful therapeutic way to limit liver inflammation and fibrosis [47]. In addition, IL18R $1^{-/}$mice displayed a less severe liver fibrotic phenotype than wild-type animals [48].

$K l r k 1$, also known as $N k g 2 d$, is an activating receptor expressed on the surface of NK cells, $\mathrm{CD}^{+} \mathrm{T}$ cells, and subsets of $\mathrm{CD}^{+} \mathrm{T}$ cells and invariant NKT cells [49]. KLRK1 mRNA increased in patients with NASH [50]. Lymphoid enhancer binding factor Lef1 is essential for the early stages of thymocyte maturation, and is involved in liver fibrosis [51, 52]. The physiological significance of miR-362-5p in the regulation of NK cell function has been studied [53]. Up-regulation of miR-362-5p is associated with hepatocellular carcinoma and inhibition of miR-362-5p dramatically decreases tumor growth and metastasis [54]. Regulation of miR-362-5p could be inferred from our results. MiR-296-5p expression is reduced in liver samples from NASH patients [55]. Here, we observed that cir_0009212 and miR-296-5ptargeted mRNAs were increased in NASH mice and that QG partially restored these alterations.

Although we obtained abundant information through ceRNA networks, and hinted at the molecular mechanisms of QG on NASH, the limitations of our study should also be noted. The MCD diet-induced mice manifested obvious hepatic steatosis and inflammation, but lacked the human metabolic profile, such as insulin resistance, and dyslipidemia. The substantial weight loss of the mice was not observed in NASH patients. In addition, since we only investigated the levels of mRNAs and not their coded proteins, a thorough and comprehensive human investigation needs to be performed to confirm the results. 

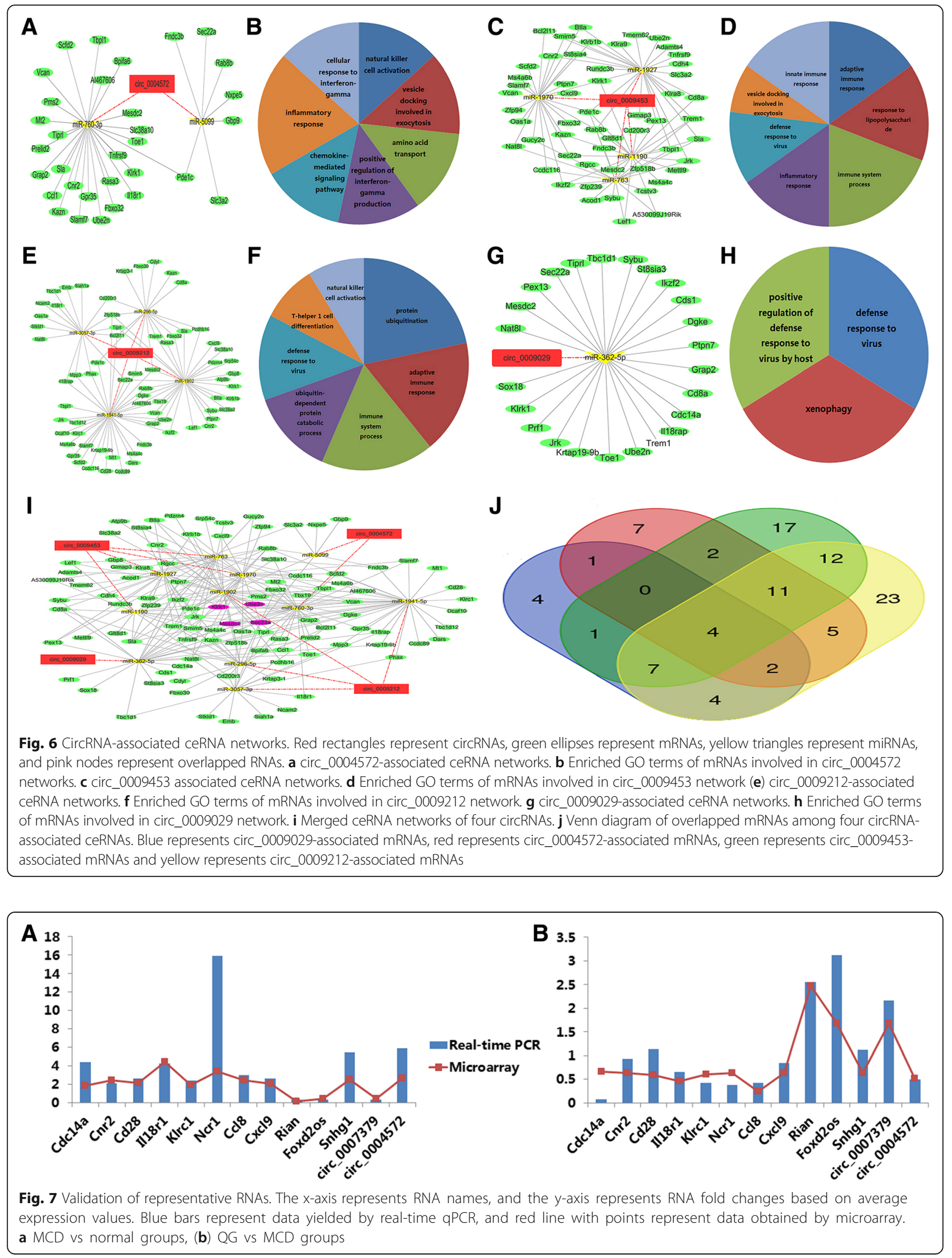


\section{Conclusions}

Our comprehensive expression profiles of mRNAs, lncRNAs and circRNAs revealed that QG might ameliorate NASH by modulating lncRNA and circRNA associated ceRNA networks. This may broaden our knowledge of QG targets and have promising therapeutic implications for NASH.

\section{Additional files}

Additional file 1: Detailed information of DEGs among groups. Sheet1: The 6193 DEGs in MCD vs normal group. Sheet2: The 514 DEGs in in QG vs MCD group. Sheet3: The overlapped 244 DEGs between MCD vs normal and QG vs MCD. (XLSX $545 \mathrm{~kb})$

Additional file 2: Detailed information of DElncRNAs among groups. Sheet1: The 5692 DEIncRNAs in MCD vs normal group. Sheet2: The 1182 DElncRNAs in in QG vs MCD group. Sheet3: The overlapped 259 DEIncRNAs between MCD vs normal and QG vs MCD. (XLSX 453 kb)

Additional file 3: Detailed information of DEcircRNAs among groups. Sheet1: The 4843 DEcircRNAs in MCD vs normal group. Sheet2: The 443 DEcircRNAs in in QG vs MCD group. Sheet3: The overlapped 98 DEcircRNAs between MCD vs normal and QG vs MCD. (XLSX 349 kb)

Additional file 4: Detailed information of GO germs and KEGG pathways. Sheet1: GO terms. Sheet2: KEGG pathways. (XLSX 64 kb)

Additional file 5: Detailed information of predicted miRNAs for DEGs, DElncRNAs and DEcircRNAs. Sheet1: The predicted miRNAs for DEGs. Sheet2: The predicted miRNAs for DElncRNAs. Sheet3: The predicted miRNAs for DEcircRNAs. (XLSX $771 \mathrm{~kb}$ )

\section{Abbreviations}

ALP: Alkaline phosphatase; ALT: Serum alanine aminotransferase; AST: Aspartate aminotransferase; ceRNAs: Competing endogenous RNAs; circRNAs: Circular RNAs; DEcircRNAs: Differentially expressed genes circRNAs; DEGs: Differentially expressed genes; DElncRNAs: Differentially expressed IncRNAs; GO: Gene ontology; KEGG: Kyoto Encyclopedia of Genes and Genomes; LDH: Lactate dehydrogenase; IncRNAs: Long non-coding RNAs; MCD: Methionine-and choline-deficient diet; MCODE: Molecular Complex Detection; miRNAs: MicroRNAs; mRNAs: Ribonucleic acids; NAFLD: Nonalcoholic fatty liver disease; NASH: Nonalcoholic steatohepatitis; ncRNAs: Non-coding RNAs; PPI: Protein-protein interaction; QG: Qiang-Gan extracts

\section{Acknowledgments}

Not applicable

\section{Authors' contributions}

$M Z$ and $M L$ contributed equally to this work; ML, LZ, YY and FL performed the majority of the experiments; $M Z$ and $L Z$ wrote the manuscript; GJ and $L Z$ designed the project, guided the whole process. All authors read and approved the final manuscript.

\section{Funding}

This work was supported by grants from the National Natural Science Foundation of China (No. 81620108030, 81774084), the Shanghai Rising Star Program (No.17QA1404000). The funding body has no role in the design of the study, collection, analysis, and interpretation of data, and in writing the manuscript.

\section{Availability of data and materials}

The datasets used and/or analyzed during the current study are available from the corresponding author on reasonable request.

\section{Ethics approval}

All animal procedures were approved by the Animal Experiment Ethics Committee of Shanghai University of Traditional Chinese Medicine.

\section{Consent for publication}

Not applicable

\section{Competing interests}

The authors declare that they have no competing interests.

\section{Author details}

${ }^{1}$ Institute of Digestive Diseases, Longhua Hospital, Shanghai University of Traditional Chinese Medicine, No.725 South Wanping Road, Shanghai 200032, China. ${ }^{2}$ School of Public Health, Shanghai University of Traditional Chinese Medicine, Shanghai 201203, China. ${ }^{3}$ Experiment Center for Science and Technology, Shanghai University of Traditional Chinese Medicine, Shanghai 201203, China.

Received: 13 January 2019 Accepted: 25 June 2019

Published online: 03 July 2019

\section{References}

1. Chalasani N, Younossi Z, Lavine JE, Diehl AM, Brunt EM, Cusi K, Charlton M, Sanyal AJ. The diagnosis and management of non-alcoholic fatty liver disease: practice guideline by the American Association for the Study of Liver Diseases, American College of Gastroenterology, and the American Gastroenterological Association. Hepatology. 2012;55(6):2005-23.

2. Baffy G, Brunt EM, Caldwell SH. Hepatocellular carcinoma in non-alcoholic fatty liver disease: an emerging menace. J Hepatol. 2012;56(6):1384-91.

3. Karbalaei R, Piran M, Rezaei-Tavirani M, Asadzadeh-Aghdaei H, Heidari MH. A systems biology analysis protein-protein interaction of NASH and IBD based on comprehensive gene information. Gastroenterology and hepatology from bed to bench. 2017;10(3):194-201

4. Takahashi Y, Sugimoto K, Inui H, Fukusato T. Current pharmacological therapies for nonalcoholic fatty liver disease/nonalcoholic steatohepatitis. World J Gastroenterol. 2015;21(13):3777-85.

5. Day CP, James OF. Hepatic steatosis: innocent bystander or guilty party? Hepatology. 1998;27(6):1463-6.

6. Tilg H, Moschen AR. Evolution of inflammation in nonalcoholic fatty liver disease: the multiple parallel hits hypothesis. Hepatology. 2010;52(5):1836-46.

7. Ratziu V, Goodman Z, Sanyal A. Current efforts and trends in the treatment of NASH. J Hepatol. 2015:62(1 Suppl):S65-75.

8. Jadeja R, Devkar RV, Nammi S. Herbal medicines for the treatment of nonalcoholic steatohepatitis: current scenario and future prospects. Evidence-based complementary and alternative medicine: eCAM. 2014; 2014:648308.

9. Li L, Zhang XJ, Lan Y, Xu L, Zhang XZ, Wang HH. Treatment of nonalcoholic fatty liver disease by Qianggan capsule. Chinese journal of integrative medicine. 2010;16(1):23-7.

10. Leti F, Legendre C, Still CD, Chu X, Petrick A, Gerhard GS, DiStefano JK. Altered expression of MALAT1 IncRNA in nonalcoholic steatohepatitis fibrosis regulates CXCL5 in hepatic stellate cells. Transl Res. 2017;190:25-39 e21.

11. Ragusa M, Barbagallo C, Brex D, Caponnetto A, Cirnigliaro M, Battaglia R, Barbagallo D, Di Pietro C, Purrello M. Molecular Crosstalking among noncoding RNAs: a new network layer of genome regulation in Cancer. Int J Genomics. 2017;2017:4723193.

12. Friedman $R C$, Farh $K K$, Burge $C B$, Bartel DP. Most mammalian mRNAs are conserved targets of microRNAs. Genome Res. 2009;19(1):92-105.

13. Lakshman R, Shah R, Reyes-Gordillo K, Varatharajalu R. Synergy between NAFLD and AFLD and potential biomarkers. Clin Res Hepatol Gastroenterol. 2015;39(Suppl 1):S29-34

14. Szabo G, Bala S. MicroRNAs in liver disease. Nat Rev Gastroenterol Hepatol. 2013;10(9):542-52.

15. Takahashi K, Yan I, Haga H, Patel T. Long noncoding RNA in liver diseases. Hepatology. 2014;60(2):744-53.

16. Atanasovska B, Rensen SS, van der Sijde MR, Marsman G, Kumar V, Jonkers I, Withoff S, Shiri-Sverdlov R, Greve JWM, Faber KN, et al. A liver-specific long noncoding RNA with a role in cell viability is elevated in human nonalcoholic steatohepatitis. Hepatology. 2017;66(3):794-808.

17. Salmena L, Poliseno L, Tay Y, Kats L, Pandolfi PP. A ceRNA hypothesis: the Rosetta stone of a hidden RNA language? Cell. 2011;146(3):353-8.

18. Cortes-Lopez M, Miura P. Emerging functions of circular RNAs. Yale J Biol Med. 2016;89(4):527-37. 
19. Zhang S, Zhu D, Li H, Li H, Feng C, Zhang W. Characterization of circRNAassociated-ceRNA networks in a senescence-accelerated mouse prone 8 brain. Mol Ther. 2017;25(9):2053-61.

20. Sui J, Xu SY, Han J, Yang SR, Li CY, Yin LH, Pu YP, Liang GY. Integrated analysis of competing endogenous RNA network revealing IncRNAs as potential prognostic biomarkers in human lung squamous cell carcinoma. Oncotarget. 2017;8(39):65997-6018.

21. Ashburner M, Ball CA, Blake JA, Botstein D, Butler H, Cherry JM, Davis AP, Dolinski K, Dwight SS, Eppig JT, et al. Gene ontology: tool for the unification of biology. The gene ontology consortium. Nat Genet. 2000;25(1):25-9.

22. Kanehisa M, Sato Y, Kawashima M, Furumichi M, Tanabe M. KEGG as a reference resource for gene and protein annotation. Nucleic Acids Res. 2016;44(D1):D457-62.

23. Wei C, Luo T, Zou S, Zhou X, Shen W, Ji X, Li Q, Wu A. Differentially expressed IncRNAs and miRNAs with associated ceRNA networks in aged mice with postoperative cognitive dysfunction. Oncotarget. 2017;8(34):55901-14.

24. Zhu M, Wang Q, Zhou W, Liu T, Yang L, Zheng P, Zhang L, Ji G. Integrated analysis of hepatic mRNA and miRNA profiles identified molecular networks and potential biomarkers of NAFLD. Sci Rep. 2018;8(1):7628

25. Wang H, Zhao YL, Xu KC. Clinical and pathological study on effects of Qianggan capsule combined lamivudine on hepatic fibrosis in patients with chronic hepatitis B. Zhongguo Zhong Xi Yi Jie He Za Zhi. 2006;26(11):978-80.

26. Liu L, Mao D, Lv J, Huang R. Meta analysis of efficacy and safety of Qianggan capsules on patients with non-alcoholic fatty liver disease. Chinese Traditional Patent Medicine. 2018;40(8):6.

27. Zheng $P$, Wang $L$, Zhang L, Liu T, Xing L, Ji G. Therapeutic effect of Qiangganjiaonang on nonalcoholic rat fatty liver by increasing the expression of liver leptin receptor and P-JAK2/P-STAT3. Chinese Journal of Integrated Traditional and Western Medicine on Digestion. 2009; 17(3):141-5.

28. Sutti S, Bruzzi S, Albano E. The role of immune mechanisms in alcoholic and nonalcoholic steatohepatitis: a 2015 update. Expert Rev Gastroenterol Hepatol. 2016;10(2):243-53.

29. Byun JS, Yi HS. Hepatic immune microenvironment in alcoholic and nonalcoholic liver disease. Biomed Res Int. 2017;2017:6862439.

30. Sun C, Liu X, Yi Z, Xiao X, Yang M, Hu G, Liu H, Liao L, Huang F. Genomewide analysis of long noncoding RNA expression profiles in patients with non-alcoholic fatty liver disease. IUBMB Life. 2015;67(11):847-52.

31. Sahu D, Hsu CL, Lin CC, Yang TW, Hsu WM, Ho SY, Juan HF, Huang HC. Co-expression analysis identifies long noncoding RNA SNHG1 as a novel predictor for event-free survival in neuroblastoma. Oncotarget. 2016;7(36):58022-37.

32. Sun $X$, Wang Z, Yuan W. Down-regulated long non-coding RNA SNHG1 inhibits tumor genesis of colorectal carcinoma. Cancer Biomark. 2017;20(1):67-73.

33. Zhang M, Wang W, Li T, Yu X, Zhu Y, Ding F, Li D, Yang T. Long noncoding RNA SNHG1 predicts a poor prognosis and promotes hepatocellular carcinoma tumorigenesis. Biomedicine \& pharmacotherapy = Biomedecine \& pharmacotherapie. 2016;80:73-9.

34. Fang $\mathrm{YL}$, Chen $\mathrm{H}$, Wang $\mathrm{CL}$, Liang L. Pathogenesis of non-alcoholic fatty liver disease in children and adolescence: from "two hit theory" to "multiple hit model". World J Gastroenterol. 2018;24(27):2974-83.

35. Li J, Zhang Z, Xiong L, Guo C, Jiang T, Zeng L, Li G, Wang J. SNHG1 IncRNA negatively regulates miR-199a-3p to enhance CDK7 expression and promote cell proliferation in prostate cancer. Biochem Biophys Res Commun. 2017; 487(1):146-52.

36. Papotto PH, Marengo EB, Sardinha LR, Goldberg AC, Rizzo LV. Immunotherapeutic strategies in autoimmune uveitis. Autoimmun Rev. 2014;13(9):909-16.

37. Poggi M, Morin SO, Bastelica D, Govers R, Canault M, Bernot D, Georgelin O, Verdier M, Burcelin R, Olive D, et al. CD28 deletion improves obesityinduced liver steatosis but increases adiposity in mice. Int J Obes. 2015; 39(6):977-85.

38. Biros E, Gabel G, Moran CS, Schreurs C, Lindeman JH, Walker PJ, Nataatmadja M, West M, Holdt LM, Hinterseher I, et al. Differential gene expression in human abdominal aortic aneurysm and aortic occlusive disease. Oncotarget. 2015;6(15):12984-96

39. Czaja AJ. Review article: chemokines as orchestrators of autoimmune hepatitis and potential therapeutic targets. Aliment Pharmacol Ther. 2014; 40(3):261-79.

40. Saeki C, Nakano M, Takahashi H, Saito S, Homma S, Tajiri H, Zeniya M. Accumulation of functional regulatory $T$ cells in actively inflamed liver in mouse dendritic cell-based autoimmune hepatic inflammation. Clin Immunol. 2010;135(1):156-66.

41. Semba T, Nishimura M, Nishimura S, Ohara O, Ishige T, Ohno S, Nonaka K, Sogawa K, Satoh M, Sawai S, et al. The FLS (fatty liver Shionogi) mouse reveals local expressions of lipocalin-2, CXCL1 and CXCL9 in the liver with non-alcoholic steatohepatitis. BMC Gastroenterol. 2013;13:120.

42. Tuo H, Wang Y, Wang L, Yao B, Li Q, Wang C, Liu Z, Han S, Yin G, Tu K, et al. MiR-324-3p promotes tumor growth through targeting DACT1 and activation of Wnt/beta-catenin pathway in hepatocellular carcinoma. Oncotarget. 2017;8(39):65687-98.

43. Cepero-Donates Y, Lacraz G, Ghobadi F, Rakotoarivelo V, Orkhis S, Mayhue M, Chen YG, Rola-Pleszczynski M, Menendez A, llangumaran S, et al. Interleukin-15-mediated inflammation promotes non-alcoholic fatty liver disease. Cytokine. 2016;82:102-11.

44. Yonekawa C, Nakae H, Zheng YJ, Wada H, Tanaka H, Tajimi K. IL-15 levels in patients with acute hepatic failure. Res Commun Mol Pathol Pharmacol. 2004;115-116:5-14.

45. Pandey A, Sahu AR, Wani SA, Saxena S, Kanchan S, Sah V, Rajak KK, Khanduri A, Sahoo AP, Tiwari AK, et al. Modulation of host miRNAs transcriptome in lung and spleen of Peste des Petits ruminants virus infected sheep and goats. Front Microbiol. 2017;8:1146.

46. Yang R, Yang F, Huang Z, Jin Y, Sheng Y, Ji L. Serum microRNA-122-3p, microRNA-194-5p and microRNA-5099 are potential toxicological biomarkers for the hepatotoxicity induced by Airpotato yam. Toxicol Lett. 2017;280:125-32.

47. Heymann F, Hammerich L, Storch D, Bartneck M, Huss S, Russeler V, Gassler N, Lira SA, Luedde T, Trautwein C, et al. Hepatic macrophage migration and differentiation critical for liver fibrosis is mediated by the chemokine receptor C-C motif chemokine receptor 8 in mice. Hepatology. 2012; 55(3):898-909.

48. Markowitz GJ, Yang P, Fu J, Michelotti GA, Chen R, Sui J, Yang B, Qin WH, Zhang Z, Wang FS, et al. Inflammation-dependent IL 18 signaling restricts hepatocellular carcinoma growth by enhancing the accumulation and activity of tumor-infiltrating lymphocytes. Cancer Res. 2016;76(8):2394-405.

49. Lanier LL. NKG2D receptor and its ligands in host defense. Cancer immunology research. 2015;3(6):575-82.

50. Kahraman A, Schlattjan M, Kocabayoglu P, Yildiz-Meziletoglu S, Schlensak M, Fingas CD, Wedemeyer I, Marquitan G, Gieseler RK, Baba HA, et al. Major histocompatibility complex class I-related chains a and B (MIC a/B): a novel role in nonalcoholic steatohepatitis. Hepatology. 2010;51(1):92-102.

51. Steinke FC, Xue HH. From inception to output, Tcf1 and Lef1 safeguard development of T cells and innate immune cells. Immunol Res. 2014; 59(1-3):45-55.

52. Desert R, Mebarki S, Desille M, Sicard M, Lavergne E, Renaud S, Bergeat D, Sulpice L, Perret C, Turlin B, et al. "Fibrous nests" in human hepatocellular carcinoma express a Wnt-induced gene signature associated with poor clinical outcome. Int J Biochem Cell Biol. 2016;81(Pt A):195-207.

53. Ni F, Guo C, Sun R, Fu B, Yang Y, Wu L, Ren S, Tian Z, Wei H. MicroRNA transcriptomes of distinct human NK cell populations identify miR-362-5p as an essential regulator of NK cell function. Sci Rep. 2015;5:9993.

54. Ni F, Zhao H, Cui H, Wu Z, Chen L, Hu Z, Guo C, Liu Y, Chen Z, Wang $X$, et al. MicroRNA-362-5p promotes tumor growth and metastasis by targeting CYLD in hepatocellular carcinoma. Cancer Lett. 2015;356(2 Pt B):809-18.

55. Cazanave SC, Mott JL, Elmi NA, Bronk SF, Masuoka HC, Charlton MR, Gores GJ. A role for miR-296 in the regulation of lipoapoptosis by targeting PUMA. J Lipid Res. 2011;52(8):1517-25.

\section{Publisher's Note}

Springer Nature remains neutral with regard to jurisdictional claims in published maps and institutional affiliations. 